\title{
Aerodynamic Sound and Flow Field Generated from Combinational Inclined Tapered Cylinder
}

\author{
Kohei Sato, Ryutaro Machida, Toshiyuki Aoki \\ Graduate School of Engineering Sources, Kyushu University, Kasuga, Japan \\ Email: 2ES11170P@s.kyushu-u.ac.jp, aoki.toshiyuki.418@m.kyushu-u.ac.jp
}

Received September 11, 2012; revised October 23, 2012; accepted November 1, 2012

\begin{abstract}
This study deals with the aerodynamic sound, especially the Aeolian tone. One of the problems with the aerodynamic sound, noise reduction along a railway line is significant engineering issue for further speed-up of a high-speed train. In particular, a pantograph is one of the main aerodynamic noise sources of a high-speed train. As one of the methods for the aerodynamic noise reduction, this study proposes the tapered cylinder by a pantograph. In this paper, the aerodynamic sound and the wake flow of the combinational inclined circular cylinder and tapered cylinder are experimentally and numerically investigated in knuckle upstream and knuckle downstream. Also, we suggest that the surface of grooves such as wood grain interacts with the aerodynamic sound generated from the model. Here, it is considered that the comparison of aerodynamic sound between the model with grooves and without grooves. Consequently, reduction of the maximum aerodynamic sound is possible for the tapered cylinder in the case of knuckle downstream. Furthermore, the noise reduction method is effective that the model surface with grooves such as wood grain.
\end{abstract}

Keywords: Aerodynamic Sound; Aeolian Tone; Inclined Tapered Cylinder; Flow Field

\section{Introduction}

This study deals with the aerodynamic sound, especially the Aeolian tone. One of the problems with the aerodynamic sound, noise reduction along a railway line is significant engineering issue for further speed-up of a highspeed train. The way of the noise reduction has strongly been required. As far as the present high-speed train is concerned, the contribution of aerodynamic sound is dominant when comparing with other kinds of the noises, such as rolling noise, concrete structure noise and so on. In particular, a pantograph is one of the main aerodynamic noise sources of a high-speed train. Therefore, researcher who develops the low noise pantograph has been continued [1-4]. The low noise pantograph is constructed to a configuration as simple as possible to reduce noise sources. The form of a pantograph should be aerodynamically-smoothed to reduce the aerodynamic sound.

The Aeolian tone generated from a circular cylinder has been widely studied by many researchers. In general, a straight circular cylinder generates narrowband peak noise. According to other researchers $[5,6]$, the tapered cylinder generates lower level of Aeolian tone than the circular cylinder. Furthermore, when the side of the large diameter of the tapered cylinder is set downstream at the inclined from -40 to 40 degrees, the Aeolian tone becomes large compared with the zero degrees $[7,8]$. It is clear that the Aeolian tone is governed with the flow fluctuation in the wake.

As one of the methods for aerodynamic noise reduction, this study proposes the tapered cylinder by a pantograph as shown in Figure 1. Thus it is important that the characteristics of the combinational inclined tapered cylinder should be made clear.

In this paper, we experimentally and numerically investigate the aerodynamic sound and the wake flow of the combinational inclined tapered cylinder and circular cylinder in knuckle upstream and knuckle downstream.

\section{Experimental Procedure}

\subsection{Test Models}

Figure 2 shows the experimental models. There are three types of the models, that is, 1) Type A for combinational inclined circular cylinder (diameter: $D=12 \mathrm{~mm}$ ); 2) Type B for combinational inclined tapered cylinder (increasing diameter to knuckle); and 3) Type $\mathrm{C}$ for combinational inclined tapered cylinder (increasing diameter from knuckle). The minimum diameter of each model is $12 \mathrm{~mm}$. When diameter is $12 \mathrm{~mm}$, it is enough to satisfy the aspect ratio for Aeolian tone generation. Here, the aspect ratio is 15 which calculated from the nozzle height and the model diameter. The tapered angle is 5 degrees, and knuckle angle is 60 degrees. The material of the 


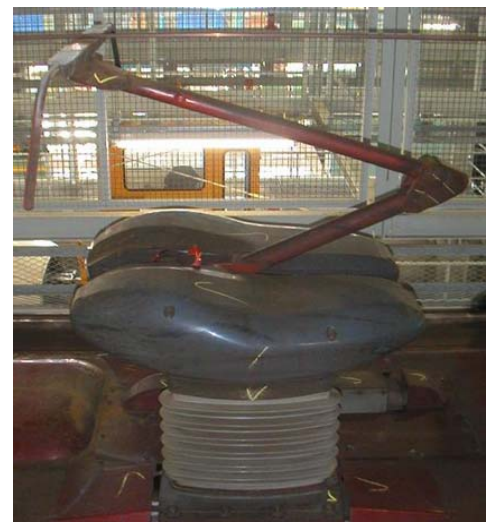

Figure 1. Photograph of the pantograph.

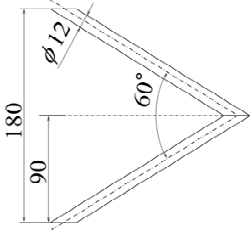

(a)

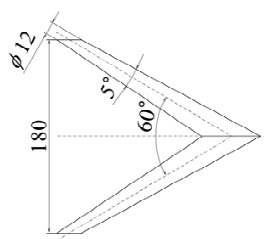

(b)

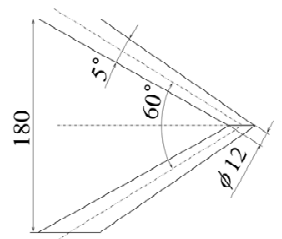

(c)
Figure 2. Schematics of the experimental models: (a) Type A; (b) Type B; (c) Type C.

models is wood which has some tiny grooves on the surface.

\subsection{Experimental Apparatus}

To measure the aerodynamic sound and the wake flow, experiment is carried out with the wind tunnel in the anechoic room. Figure 3 shows the experimental apparatus. The nozzle size is $180 \mathrm{~mm} \times 360 \mathrm{~mm}$ and flow velocity range is from 0 to $33 \mathrm{~m} / \mathrm{s}$. In order to satisfy both dimensionality and free field acoustically, acoustic transmission board is installed at top and bottom wall with the wind tunnel. The models are located at $170 \mathrm{~mm}$ and 326 $\mathrm{mm}$ from the nozzle exit to knuckle in knuckle upstream and knuckle downstream, respectively.

The aerodynamic sound is measured by $1 / 2$ inch microphone. The microphone is 3 positions which are located at zero degrees (Position 1), 45 degrees (Position 2) and 90 degrees (Position 3) from horizontal flow direction, and radial distance of $1000 \mathrm{~mm}$ from the knuckle of the models as shown in Figure 3. The data recorded by FFT analyzer. The number of sampling data is 16384 with a sampling frequency of $20 \mathrm{kHz}$ for power spectral averaging procedure of 50. The filtering of the aerodynamic sound is A-weighted.

The flow velocity in the wake is measured by a hot wire anemometer as shown in Figure 4. The sampling frequency is $10 \mathrm{kHz}$ for measuring time of 5 seconds. The coordinate system is defined for the measurement, in which the $x$-axis, the $y$-axis and the $z$-axis are set in the

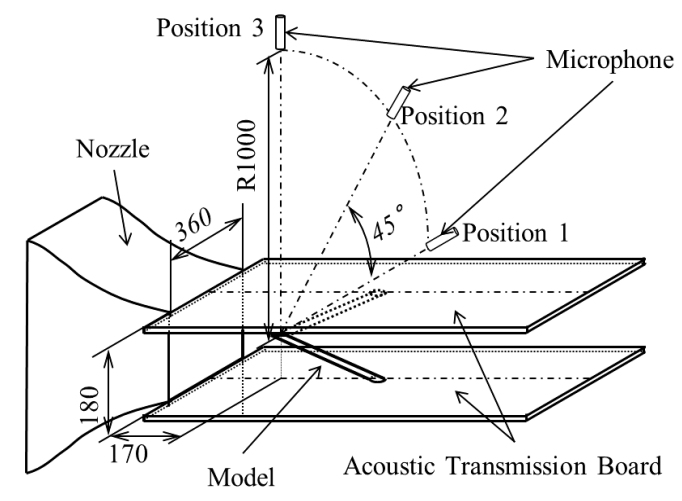

Figure 3. Experimental apparatus.

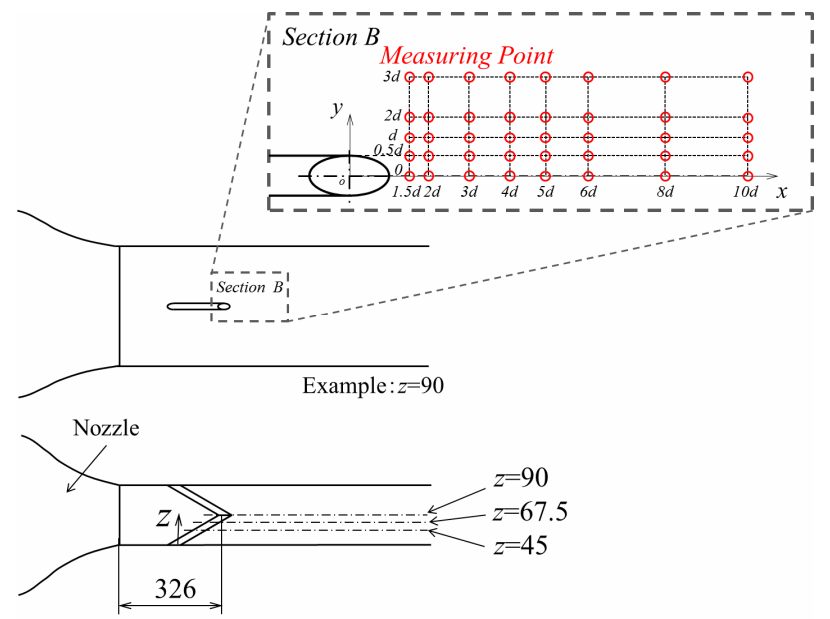

Figure 4. Velocity measurement position.

flow direction, the vertical and the lateral direction, respectively. The origin are taken at the center on the $x y$ plane of the models at $z=45,67.5,90 \mathrm{~mm}$. The data is measured at 8 positions in the $x$-axis direction $x / D=1.5$, $2,3,4,5,6,8$ and 10,5 positions in the $y$-axis direction $y / D=0,0.5,1,2$ and 3,3 positions in the $z$-axis direction $z=45,67.5,90 \mathrm{~mm}$. In the discussions chapter, the averaged velocity and the power spectra density is used.

\subsection{Visualization of Streamline by Numerical Analysis}

In order to investigate flow field around the models, we analyzed by the numerical simulation. The CFD analysis with ANSYS FLUENT is used in the numerical simulation. The numerical condition is listed in Table $\mathbf{1}$.

\subsection{Influence of the Model Surface with Grooves}

As mentioned above, it was used in the present experiment that the material of the models which has some tiny grooves on the surface (unpainted model). We suggest that the surface of grooves interacts with aerodynamic sound generated from the models. Here, it is considered 
that the comparison of the aerodynamic sound between the unpainted model and the painted model. The painted model does not almost have grooves on surface compared with the unpainted model as shown in Figure 5.

\section{Results and Discussions}

In this chapter, we experimentally discuss the aerodynamic sound and the wake flow of the combinational inclined tapered cylinder in knuckle upstream and knuckle downstream.

\subsection{Aerodynamic Sound Characteristics}

Figure 6 shows the experimental results of aerodynamic sound of these models at the flow velocity $30 \mathrm{~m} / \mathrm{s}$ which are in knuckle upstream and downstream. The experimental results is $1 / 3$ octave-band level of aerodynamic sound when the microphone is located at position 1. As shown in Figure 6, BGN is the background noise which measured without the model in the wind tunnel. Also, knuckle upstream and knuckle downstream means knuckle forward direction and backward direction, respectively.

Figure 6(a) shows the aerodynamic sound in knuckle upstream. The combinational inclined tapered cylinder for type $\mathrm{B}$ and type $\mathrm{C}$ generates clearly higher level of broadband noise than the combinational inclined circular cylinder for type A. This result is caused by diameter of the tapered cylinder is larger than that of the circular

Table 1. Numerical condition.

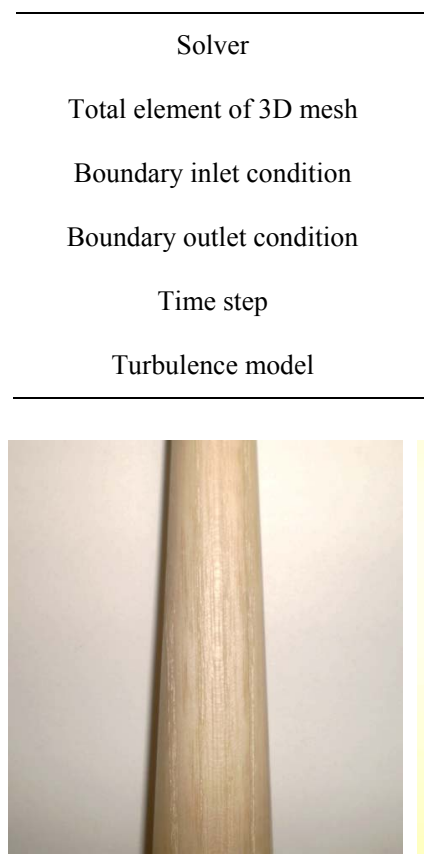

(a)
Pressure-Based Coupled Solver

\section{About 2 million}

Flow Velocity: $30 \mathrm{~m} / \mathrm{s}$

Static Pressure: 1 atm

$$
4.7 \times 10^{-3} \mathrm{sec}
$$

$$
k-\varepsilon
$$

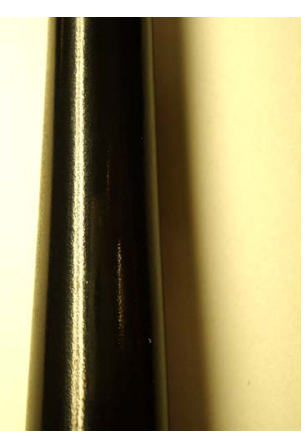

(b)
Figure 5. Photograph of the unpainted model and the painted model: (a) Unpainted model; (b) Painted model. cylinder. Figure 6(b) shows type B generates lower level of narrowband noise from 200 to $800 \mathrm{~Hz}$ than type $\mathrm{A}$ in knuckle downstream. Here, the maximum aerodynamic sound is presented at frequency about $250 \mathrm{~Hz}$ for type A. If Strouhal number is 0.2 , the vortex shedding frequency is calculated $250 \mathrm{~Hz}$. Thus, it indicates that vortex sound is suppressed by the tapered cylinder. This result suggests that the tapered cylinder is effective to reduce the maximum aerodynamic sound in knuckle downstream. In order to investigate the mechanism of noise reduction, we measured the flow fluctuation of most effective type $\mathrm{B}$ in knuckle downstream.

\subsection{Wake Flow Characteristics}

Figure 7 shows the averaged flow velocity distributions in the wake generated from type A and type B at $z=90$ and $z=45$ for flow velocity $30 \mathrm{~m} / \mathrm{s}$. The horizontal and vertical axes represent the $x$-axis position [mm] and the $y$-axis position [mm], respectively. As shown in Figure 7(a-1), the velocity of type A decreases in the widely region at $z=90$. The low velocity region at $z=45$ is smaller than ones at $z=90$ as shown in Figure 7(a-2).

In contrast, the low velocity region of type $B$ at $z=90$ is larger than that of type $A$ at $z=90$ as shown in Figure $\mathbf{7 ( b - 1 )}$. The low velocity region of type $B$ at $z=45$ is

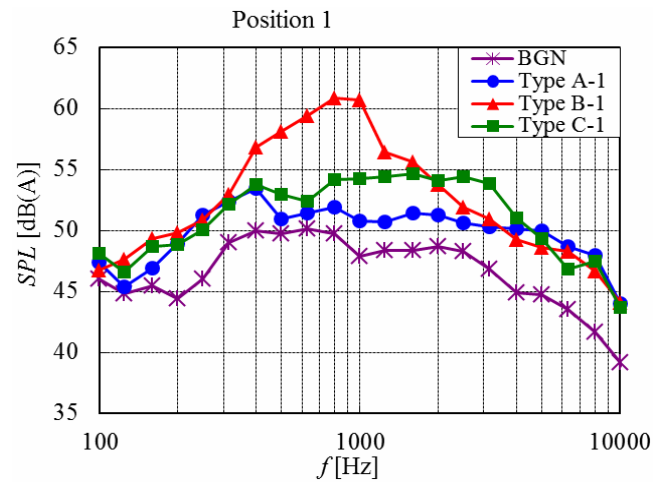

(a)

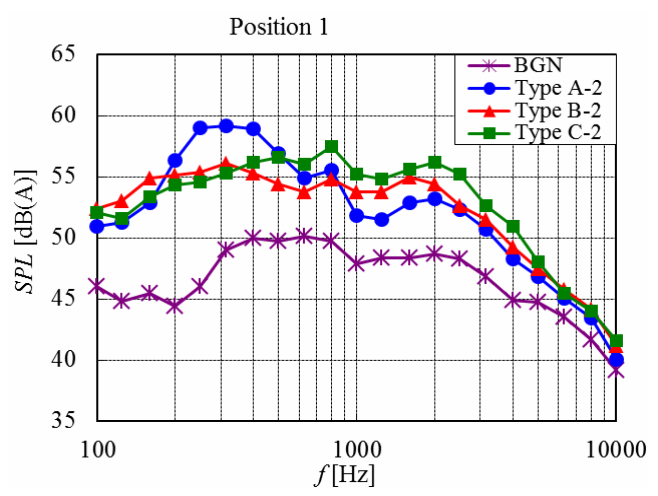

(b)

Figure 6. The aerodynamic sound characteristics: (a) Knuckle upstream; (b) Knuckle downstream. 
higher value than that of type $\mathrm{A}$ as shown in Figure 7(b-2). It suggested that the inflow from axial direction become stronger by the tapered effect. Figure 8 shows the power spectrum density (PSD) distributions of the flow fluctuation in the wake generated from type A and type $B$ at $z=90$ and $z=45$. The PSD consists of the average PSD value from 0 to $1000 \mathrm{~Hz}$ at each measurement. For type A, PSD is large in the region at both $z=90$ and $z=45$. On the contrary, a comparatively high PSD region is located at $z=90$ for type B. The PSD of type B becomes lower than that of type $A$ at $z=45$. Here, type $B$ generates the non-peak level and broadband noise. Thus, it suggested that the various scale vortexes is main noise sources. For type B, vortex generation tends to concentrate at $z=90$ on knuckle by tapered effect.

\subsection{Time-Averaged Streamline by CFD}

Figure 9 shows the CFD results of time-averaged streamline around the models for flow velocity $30 \mathrm{~m} / \mathrm{s}$ in knuckle

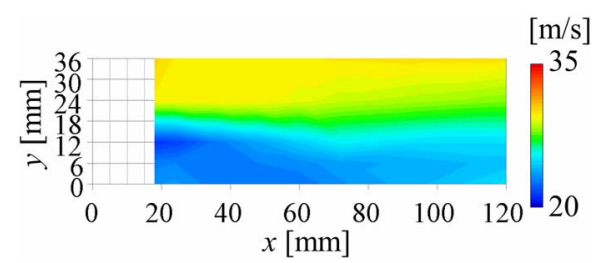

(a-1)

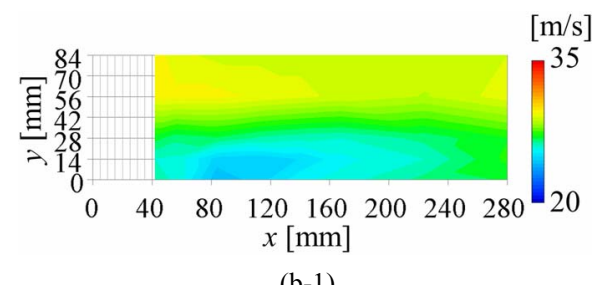

(b-1) downstream. Figures 9(a) and (b) show that the streamline of type B concentrates on a knuckle compared with that of type A. In contrast with these result, Figure 9(c) seem to be widely separated flow from inclined part of type C. It is considered that since this separated flow generates vortex of noise source.

\subsection{Comparison of the Unpainted Model and the Painted Model}

Figures 10 and 11 show the measurement result of the aerodynamic sound and the PSD distribution for the painted model of type B in knuckle downstream. Comparison with the unpainted model, the aerodynamic sound clearly increased in the broadband. Also, Figure 11(a) shows that high PSD region decreases at $z=90$. At $z=45$, high PSD region is located at $y=10$ as shown in Figure 11(b). It suggested that the separated flow widely generates in the wake by the surface flow separation changes from turbulence to laminar when the model surface

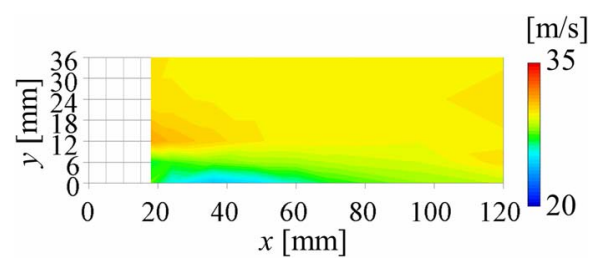

$(\mathrm{a}-2)$

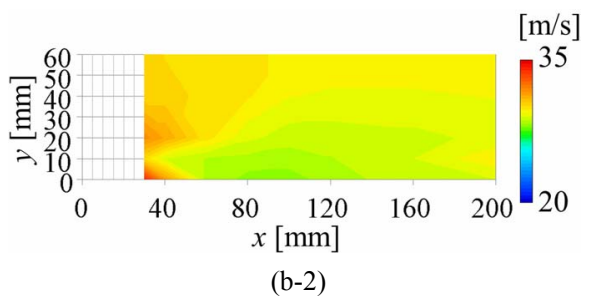

Figure 7. The averaged flow velocity distributions in the wake of type $A$ and type $B:(a-1) z=90$, type A; (a-2) $z=45$, type A; (b-1) $z=90$, type B; (b-2) $z=45$, type B.

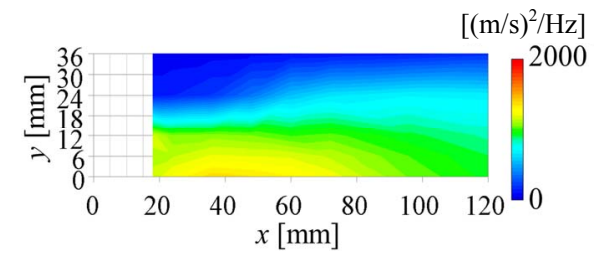

(a-1)

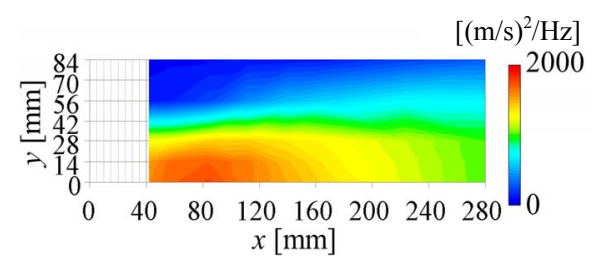

(b-1)

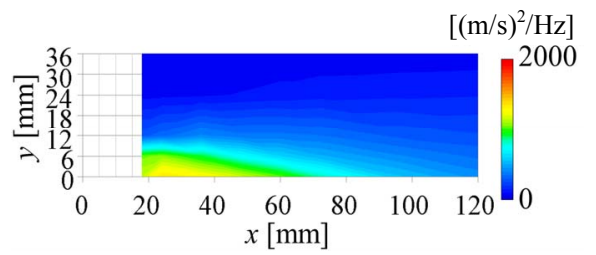

(a-2)

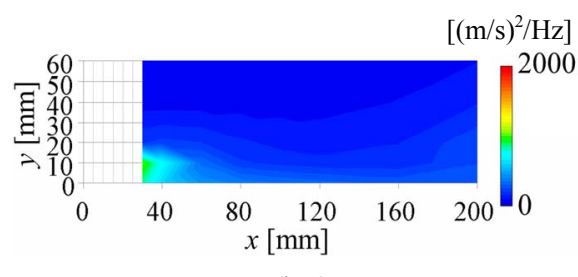

(b-2)

Figure 8. The power spectrum density distributions in the wake of type $A$ and type $B$ : (a-1) $z=90$, type A; (a-2) $z=45$, type A; (b-1) $z=90$, type B; (b-2) $z=45$, type B. 


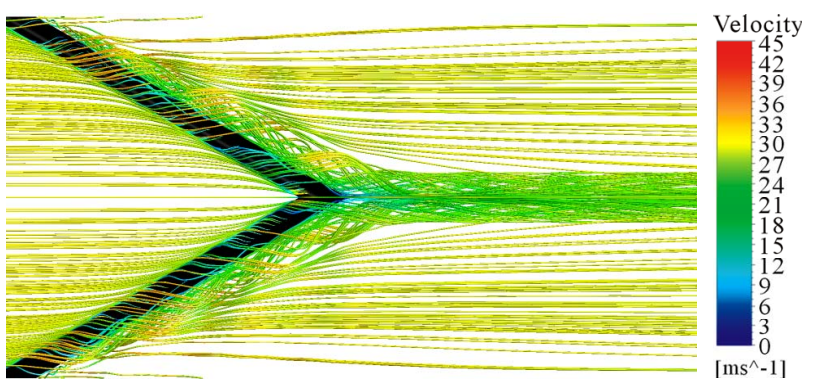

(a)

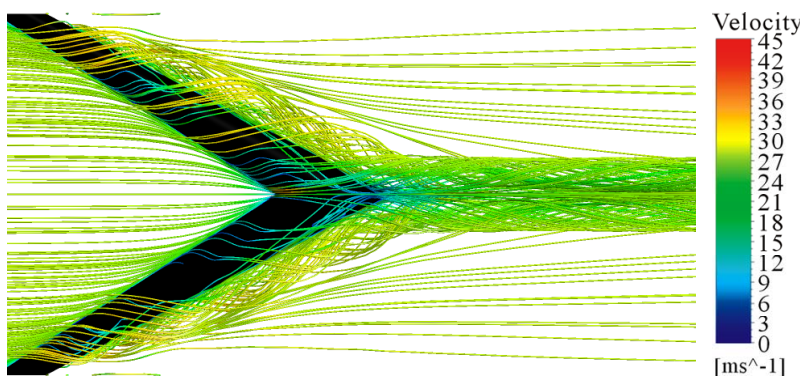

(b)

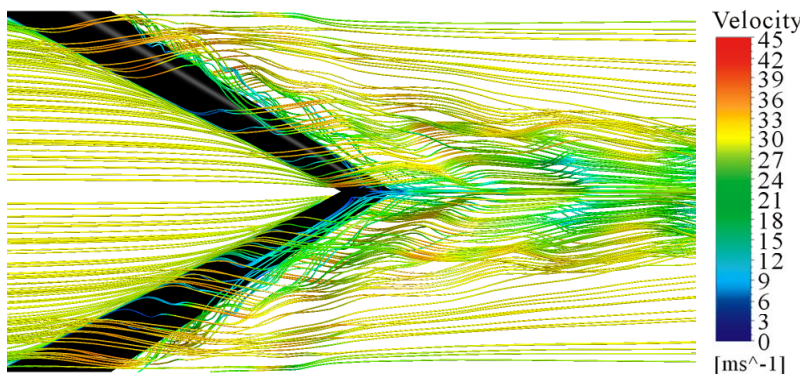

(c)

Figure 9. CFD results of time-averaged streamline around the models: (a) Type A; (b) Type B; (c) Type C.

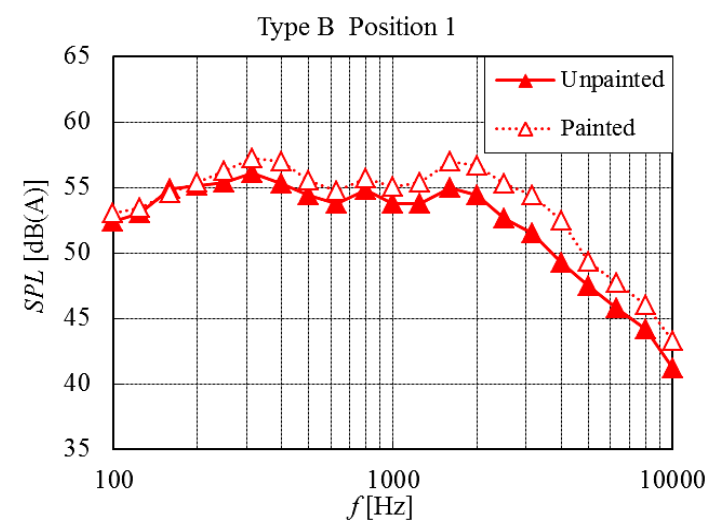

Figure 10. Comparison of the aerodynamic sound of type $B$ for the unpainted model and the painted model.

changes from with grooves to without grooves. Furthermore, it suggested that strong axial flow decreases by separated flow from inclined model surface. Thus, high aerodynamic sound is caused by widely separated flow generation of noise source.

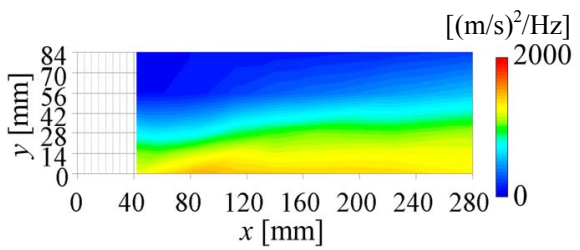

(a)

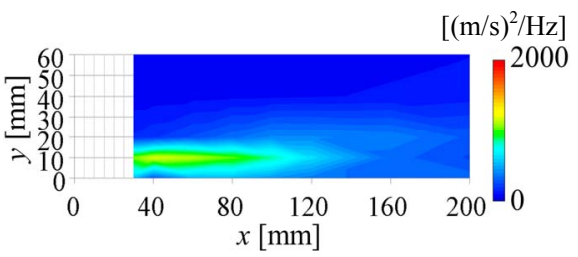

(b)

Figure 11. The power spectrum density distributions in the wake of type $B$ in the painted model: (a) PSD, $z=90$; (b) PSD, $z=45$.

\section{Conclusions}

This paper described the aerodynamic sound and the flow field of the combinational inclined circular cylinder and tapered cylinder by a pantograph.

1) In the case of knuckle upstream, the combinational inclined tapered cylinder is generated higher level of broadband noise than the combinational inclined circular cylinder;

2) In the case of knuckle downstream, the maximum aerodynamic sound of the combinational inclined tapered cylinder is suppressed in narrowband. Besides, the wake flow tends to concentrate the vortex of noise source on a knuckle;

3) When the model surface is painting in order to without grooves from with grooves, the aerodynamic sound clearly increased in the broadband. This is due to widely separated flow generation of noise source.

Consequently, reduction of the maximum aerodynamic sound is possible for the tapered cylinder in the case of knuckle downstream. Furthermore, the noise reduction method is effective that the model surface with the grooves such as wood grain.

\section{REFERENCES}

[1] T. Sueki, M. Ikeda and T. Takaishi, "The Aerodynamic Noise Reduction by Porous Materials and Application to a Current Collector," RTRI Report, Vol. 22, No. 5, 2008, pp. 26-31.

[2] Y. Ijichi, T. Odo, N. Kondoh and T. Aoki, "Sound Characteristic of Aerodynamic Noise Generated from Pantograph on a High-Speed Train," Journal of JSME, Vol. 61, No. 85-2, 2008, pp. 19-20.

[3] M. Ikeda and T. Mitsumoji, "Method to Evaluate Structure of Low-Frequency Aerodynamic Noise Source Generated by Shinkansen Pantograph," RTRI Report, Vol. 21, 
No. 12, 2007, pp. 47-52.

[4] K. Shibata, M. Hirai, T. Hariyama and Y. Sasaki, "Development of Low Noise Pantograph Adopted to the Superexpress Hayate," Mitsubishi Heavy Industries Technical Review, Vol. 40, No. 3, 2003, pp. 154-157.

[5] K. Saito, H. Fujita and Y. Suzuki, "Experimental Study of the Characteristics of the Aeolian Tone and the Wake structures Generated from Tapered Circular Cylinders," Journal of JSME Fluid Engineering Conference 2010, Vol. 1006, No. 88, 2010, pp. 271-272.

[6] J. Fukutomi, Y. Nakase and M. Takikawa, "Vortex Wake from Tapered and Stepped Circular Cylinders in a Uni- form Flow," Journal of JSME, Vol. 59, No. 92, 1993, pp. 684-690.

[7] T. Tsuchida, H. Hayashi, Y. Kodama, T. Fukano and S. Sasaki, "Relationship between Aeolian Tone and Wake of Inclined Tapered Cylinder," Journal of JSME Environment Engineering Symposium 2006, Vol. 112, No. 16, 2006, pp. 65-68.

[8] Y. Haramoto, K. Matsuzaki, M. Munekata and H. Ohba, "An Experiment and Numerical Simulation of Aerodynamic Sound Generated from Inclined Circular Cylinder," Journal of the Acoustical Society of Japan, Vol. 301, No. 58, 2002, pp. 282-288. 